\section{The adjacency principle and induced movement*}

\author{
WALTER C. GOGEL and MICHAEL KOSLOW \\ University of California, Santa Barbara, California 93106
}

The apparent movement of a stationary point of light was investigated as a function of the apparent distance of the point of light with respect to the inducing frame or frames. When two frames were presented simultaneously, they were at different distances and physically moved at opposite phase in frontoparallel planes. When one frame was presented alone, it was positioned at different times at each of the two distances. The point of light was presented stereoscopically at the distance of either the near or far frame or midway in depth between these distances. With the single frame, it was found that the magnitude of the induced movement decreased as the point of light was increasingly far in front of a frame but decreased less or remained approximately constant for distances behind a frame. With the two frames presented simultaneously, it was found that as the depth between a particular frame and the point of light decreased, the contribution of that frame to the induced movement increased. The results illustrate the interaction of perceptions, in this case perceived depth and perceived motion, and are consistent with the adjacency principle.

A stationary point of light that is viewed in a moving frame will appear to move in a direction opposite to the direction of the frame movement. The apparent movement of the physically stationary point of light is an example of induced motion (Duncker, 1939). It had been found that the reported magnitude of the induced motion can vary as a function of the apparent depth displacement of the light from the frame with this displacement produced by stereoscopic cues (Gogel \& Koslow, 1971). Specifically, the magnitude of induced motion usually decreased for a stereoscopic displacement of the point of light in front of the frame, with little or no decrease as the point of light was displaced in stereoscopic depth behind the frame. These results were explained in terms of the combined effects of two factors. One factor is the tendency for the magnitude of the induced motion to be consistent with the size-distance invariance hypothesis. It is as though the magnitude of the induced motion simulated a visual angle, with the apparent extent of the movement increasing with the apparent distance of the point of light from the $O$. The other factor is the adjacency principle, which states that the effectiveness of cue relations between objects decreases as a function of the directional or depth separation of the objects (Gogel, 1970). It would be expected from the adjacency principle that the magnitude of the induced motion would decrease as the point of light was displaced in

*This investigation was supported by U.S. Public Health Service Research Grant No MH 15651 from the National Institute of Mental Health. perceptually) at different distances moved back and forth (right and left), with the movement of the two frames out of phase. That is, when the one frame was moving to the left, the other frame was moving to the right, etc. A point of light was positioned at the distance of either frame or midway in depth between the frames. Under these conditions the induction effects of the two frames upon the point of light should be in opposite directions. According to the adjacency principle, the contribution of a particular frame to the apparent movement of the point of light should increase as the point of light is positioned increasingly close to that frame in distance and increasingly far from the other frame.

\section{EXPERIMENT}

Apparatus

A device consisting of two synchronous constant-speed motors, gears, microswitches, and a chain drive was constructed for laterally moving luminous square frames back and forth at either or both a near (3 ft) or far $(6 \mathrm{ft})$ distance from 0 , with the velocity at a particular distance constant. The movement of all of the frames was perpendicular to the median plane of $\mathrm{O}$, with instantaneous reversal in direction at the right and left extents of movement. The center of a frame was always at the height of $O$ 's eyes. Four frames were constructed, with a particular frame designed to be used at only one of the two distances and to subtend one of two visual angles at that distance. The distance positions and sizes of the four frames are schematically illustrated in Fig. 1. A small frame, $\mathrm{S}$ (3.5 in. square with 0.5 -in. border), was constructed to be used only at the near distance, $\mathrm{N}$. This frame-distance combination is $S_{\mathrm{N}}$ in Fig. 1. A large frame, L (23.2 in. square with 1.0-in. border), was

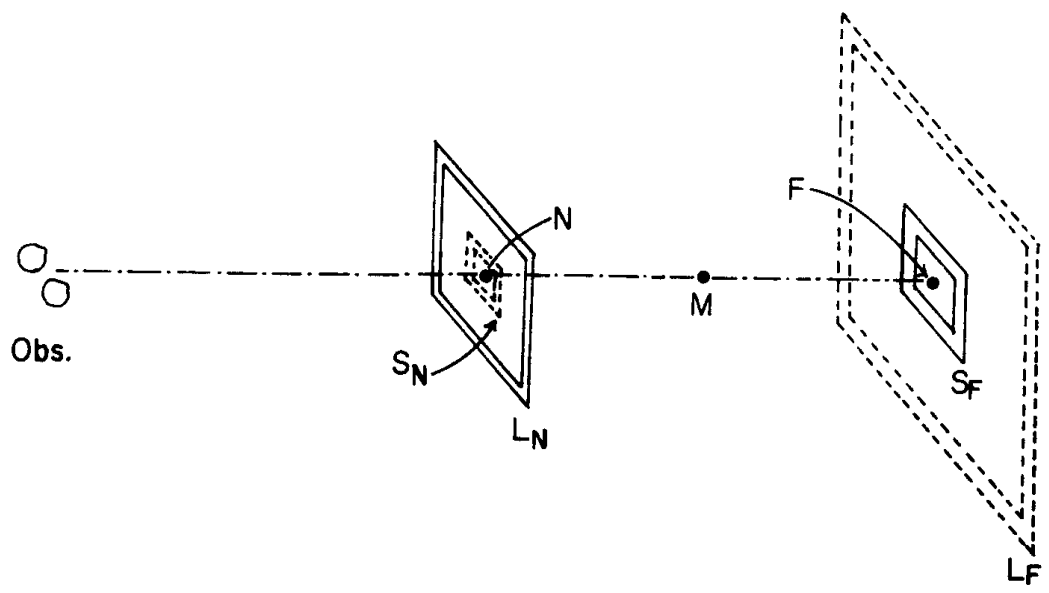

Fig. 1. Schematic perspective drawing of the four frame sizes and the distances at which they were presented in the experiment. 
constructed to be used only at the far position, F. This frame-distance combination is $L_{F}$ in Fig. 1. A small frame, $S$ ( 7 in. square with 1 -in. border), was constructed to be used only at the far position, F. This frame-distance combination is $S_{F}$ in Fig. 1. A large frame, $L$ (11.6 in. square with 0.5 -in. border), was constructed to be used only at the near position, $\mathrm{N}$. This frame-distance combination is $\mathrm{L}_{\mathrm{N}}$ in Fig. 1. These four frame-distance combinations were presented singly (one at a time) or two frames were presented simultaneously in pairs. When presented simultaneously in pairs, only two of the four possible pairs were used. These two pairs were either $S_{N}, L_{F}$ (illustrated by the dotted lines in Fig. 1), or $\mathrm{L}_{\mathrm{N}}, \mathrm{S}_{\mathrm{F}}$ (illustrated by the solid lines in Fig. 1). Since the visual angle of $S_{F}$ was equal to that of $S_{N}$ and the visual angle of $L_{N}$ was equal to that of $\mathrm{L}_{\mathrm{F}}$, it follows that the sizes on the eye of the $S_{N}, L_{F}$ pair of frames was the same as that of the $\mathrm{L}_{\mathrm{N}}, \mathrm{S}_{\mathrm{F}}$ pair of frames. The only stimulus difference between the situations involving $S_{N}, L_{F}$ and $S_{F}, L_{N}$ was that in the former situation the larger (outer) frame was at the greater $(6 \mathrm{ft})$ distance, with the smaller (inner) frame at the lesser $(3 \mathrm{ft}$ ) distance, and in the latter situation the distance positions were reversed. Whenever a pair of frames $w$ as presented simultaneously, the direction of the movement of the far frame was always opposite to the direction of movement of the near frame. The frame at the one distance was always moving to $O$ 's left whenever the frame at the other distance was moving to O's right. In order to avoid one frame obscuring the other in the simultaneous presentations during any portion of the motion, the linear extent of the movement of the frames was small. At the near and far positions the frames moved through a total right-to-left distance of .38 and .77 in., respectively, with 1 sec always required to complete a cycle (a single back-and-forth movement) for each frame at either distance. The frames were constructed from strips of electroluminescent tape (Sylvania white panelescent tape) and had a brightness of .32 fL.

The observation position was located in a booth which was totally dark except between trials and contained a chin rest and a binocular eyepiece with artificial pupils of $1 \mathrm{~mm}$ diam. The distance between the artificial pupils was adjusted for the interpupillary distance of $\mathrm{O}$ prior to $O$ 's participation in the experiment. The viewing was controlled by a shutter operated by the $E$. The artificial pupils were located very close to the cornea, with a resulting visual field of about $36 \mathrm{deg}$.

A single point of light $(1 \mathrm{~mm}$ in diam) was located at $3,4.5$, or $6 \mathrm{ft}$ from 0 . These three alternate distance positions of the point of light are labeled $N, M$, and $F$, respectively, in Fig. 1. The point of light was always physically stationary and was located in direction in the center of the frames for either the single- or the two-frame situations when the frames were symmetrically positioned with respect to $O$ 's median plane. Induced movement resulting from the moving frames would make the point of light appear to move back and forth laterally. One task of $O$ 's was to indicate the phase of the induced movement of the point of light. In order to permit $E$ to interpret $O$ 's response to phase, an indicator (an arrow visible only to E) lighted whenever the frame at the near position was moving toward the left (or conversely, whenever the frame in the far position was moving to the right). This device enabled $E$ to determine whether the reports of $\mathrm{O}$ as to the direction of the apparent motion of the point of light was in phase with or $180 \mathrm{deg}$ out of phase with the direction of movement of a frame at either the near or far position. The $\mathrm{O}$ and $\mathrm{E}$ communicated with each other by means of a speaker system. No stimuli were visible thoughout the experiment except the single frame or the simultaneously presented frames and the point of light. The remainder of the visual field was totally dark.

\section{Subjects}

Forty-eight students from an introductory course in psychology participated in this study. All Os had a stereoacuity of at least $22.08 \mathrm{sec}$ of arc, as measured with a Keystone Orthoscope, and were naive as to the purpose of the experiment.

\section{Procedure}

Each of the three distance positions of the point of light was presented with either one frame alone or two frames simultaneously. The stimulus situations in which only a single frame was presented were: (1) $S_{N}$, small frame near, (2) $S_{F}$, small frame far, (3) $\mathrm{L}_{\mathrm{N}}$, large frame near, and (4) $\mathrm{L}_{\mathrm{F}}$, large frame far. The situations in which the two frames were presented simultaneously were: $(5) \mathrm{S}_{\mathrm{N}}, \mathrm{L}_{\mathrm{F}}$, small frame near, large frame far; and (6) $\mathrm{L}_{\mathrm{N}}, \mathrm{S}_{\mathrm{F}}$, large frame near, small frame far. Each $O$ was presented with each of the above six situations and each of the three distances of the point of light was used with each situation. The order in which the six different situations was presented and the order in which the three distance positions of the point of light was used with each situation was varied systematically between Os. All Os were dark adapted for $30 \mathrm{sec}$ before beginning the experiment, and between trials an overhead light was turned on in the observation booth (with the observation shutter closed).

On each presentation of a frame-distance configuration the $O s$ had several tasks. The first task was to report the direction of apparent movement of the point of light. With half the Os this was accomplished by saying "left" when the point of light appeared to be moving to the left. With the remaining $O s$, the task was to say "right" when the point of light appeared to be moving to the right. It was found that this method permitted $E$ to determine readily whether the direction of the induced motion was coincident with or opposite to the direction of the motion of a frame at a particular distance. Before asking for the direction of movement, $E$ ascertained that $O$ saw the point of light as moving back and forth. O's second task was to report the extent of the apparent movement of the point of light in feet or inches or in some combination of feet and inches. Because of the small magnitudes of the frame movements, when induced movement was seen, this report was almost always given in inches. O's third task was to report the apparent distance of the point of light from himself, again in feet or inches or in some combination of both. Finally, after completing these tasks in the above order for a particular frame position with each of the three positions of the point of light, $\mathrm{O}$ was asked to report the apparent distance of the frame from himself. During each of these tasks, $O$ was asked to fix his gaze on the point of light.

\section{Results}

Presentation of single frame. The average and median reported extents of the induced movement of the point of light for the presentations of a single frame are given in Table 1 . Since the distributions of results were sometimes skewed, the medians, rather than the means, were considered to best represent the data. Included in Table 1 are the average ranks necessary to compute $\chi_{r}^{2}$ for the Friedman two-way analysis of variance (Siegel, 1956), with the value of $\chi_{r}^{2}$ and the significance level also included. If the induced motion of the point of light is determined by a particular frame, the induced motion will be 180 deg out of phase with the physical motion of that frame. In the case in which only one frame was present (Table 1), whenever this result occurred it was given a 
Table 1

Means, Medians, and Standard Deviations in Inches of Induced Movement of a Point of Light at the Near (N), Middle (M), and Far (F) Distance with One Frame Presented at a Time at Either the Near or Far Distance*

\begin{tabular}{|c|c|c|c|c|c|c|}
\hline & \multicolumn{3}{|c|}{$\begin{array}{c}\text { Large Frame Near }\left(\mathrm{L}_{\mathrm{N}}\right) \\
\text { Distance of Point of Light }\end{array}$} & \multicolumn{3}{|c|}{$\begin{array}{c}\text { Large Frame Far }\left(\mathrm{L}_{\mathrm{F}}\right) \\
\text { Distance of Point of Light }\end{array}$} \\
\hline & $\mathrm{N}$ & $\mathbf{M}$ & $\mathbf{F}$ & $\mathbf{N}$ & $\mathbf{M}$ & $\mathbf{F}$ \\
\hline Mean & +1.23 & +1.04 & +1.89 & +0.72 & +0.47 & +1.04 \\
\hline Median & +0.50 & +0.50 & +0.50 & +0.33 & +0.50 & +0.88 \\
\hline SD & 3.40 & 3.43 & 8.50 & 1.71 & 0.38 & 1.71 \\
\hline Number of + & 41 & 40 & 43 & 39 & 37 & 40 \\
\hline Number of - & 0 & 0 & 0 & 0 & 0 & 0 \\
\hline Number of 0 & 7 & 8 & 5 & 9 & 11 & 8 \\
\hline \multirow[t]{4}{*}{ Average Rank } & 2.30 & 1.72 & 1.98 & 1.57 & 1.88 & 2.55 \\
\hline & \multicolumn{3}{|c|}{$x^{2} x=8.1, p<.02$} & \multicolumn{3}{|c|}{$x^{2} \mathrm{r}=24.0, \mathrm{p}<.001$} \\
\hline & \multicolumn{3}{|c|}{$\begin{array}{c}\text { Small Frame Near }\left(\mathrm{S}_{\mathrm{N}}\right) \\
\text { Distance of Point of Light }\end{array}$} & \multicolumn{3}{|c|}{$\begin{array}{c}\text { Small Frame Far }\left(\mathrm{S}_{F}\right) \\
\text { Distance of Point of Light }\end{array}$} \\
\hline & $\mathbf{N}$ & $\mathbf{M}$ & $\mathrm{F}$ & $\mathbf{N}$ & M & $\mathbf{F}$ \\
\hline Mean & +0.83 & +0.45 & +0.66 & +0.48 & +0.90 & +2.29 \\
\hline Median & +0.50 & +0.25 & +0.25 & +0.25 & +0.29 & +0.50 \\
\hline SD & 1.12 & 0.69 & 1.76 & 0.98 & 1.65 & 8.59 \\
\hline Number of + & 30 & 30 & 28 & 26 & 30 & 30 \\
\hline Number of - & 0 & 0 & 1 & 2 & 1 & 1 \\
\hline Number of 0 & 18 & 18 & 19 & 20 & 17 & 17 \\
\hline \multirow[t]{2}{*}{ Average Rank } & 2.38 & 1.79 & 1.83 & \multirow{2}{*}{\multicolumn{3}{|c|}{$\chi^{2} r=19.3, p<.001$}} \\
\hline & \multicolumn{3}{|c|}{$\chi^{2} x=10.1, p<.01$} & & & \\
\hline
\end{tabular}

*A positive or negative sign for the mean or median indicates that the induced move. ment of the point of light was $180 \mathrm{deg}$ out of phase or in phase, respectively, with the physical movement of the frame.

positive sign. An apparent motion in phase with the physical motion of the single frame is not the expected result and is given a negative sign in Table 1 . A zero value in Table 1 indicates no apparent motion of the point of light, i.e., the point of light appeared to be stationary. In computing the average ranks, the three reports of the extent of the apparent motion of the point of light from each $O$ for the three different distances $(N, M, F)$ of the point of light were ranked from 1 to 3 , with the smallest magnitude of induced movement receiving the rank of 1 . In the few instances in which the negative values occurred when a single frame was used, the largest negative value was given the rank of 1 . Thus, the greater the number of the average rank, the greater was the apparent movement determined by the frame. The number of,+- , and 0 values obtained for each condition also are listed in Table 1.

Simultaneous presentations of two frames. The results from the situations in which the two frames were presented simultaneously, with one frame at the 3 -ft distance and the other at the 6-ft distance, are shown in Table 2. If the far frame determined the direction of the induced motion, i.e., if the induced motion was $180 \mathrm{deg}$ out of phase with the far frame (in phase with the near frame), the report was given a positive $(+)$ value in Table 2. If the near frame determined the direction of the induced motion, i.e., if the induced motion was $180 \mathrm{deg}$ out of phase with the near frame (in phase with the far frame), the report was given a negative $(-)$ sign in Table 2. Thus, a positive mean or median indicated that the far frame determined the direction of the induced motion and a negative mean or median indicated that the near frame determined the direction of the induced motion. In ranking the scores from each $\mathrm{O}$ obtained from the three distance positions of the point of light $(\mathrm{N}, \mathrm{M}, \mathrm{F})$, the sign of the score was taken into consideration. For example, the most negative of the three scores from an $O$ for a particular situation $\left(\mathrm{L}_{\mathrm{N}}, \mathrm{S}_{\mathrm{F}}\right.$ or $\mathrm{S}_{\mathrm{N}}, \mathrm{L}_{\mathrm{F}}$ ) was given a rank of 1 with the most positive of his three or near frame, respectively. scores given a rank of 3 . Thus, the greater the average rank in Table 2, the more the induced motion was determined by the far frame; the less the average rank in Table 2 , the more the induced motion was determined by the near frame. The resulting average ranks and the $x_{r}^{2}$ obtained from the Friedman two-way analysis of variance are also shown in Table 2. All standard deviations in Table 2 are arbitrarily listed as positive.

Table 3 gives the results in feet from the reports of the distance of the point of light and the frames averaged over the indicated frame-distance combinations. From the median results of Table 3 , the point of light and the frames usually appeared at about the same distance when they were physically at the same distance. Also, from the results of Table 3 , positioning the point of light at the middle distance, i.e., behind or in front of a frame, resulted in the point of light appearing behind or in front of that frame. The general tendency shown by the medians for the verbal reports to underestimate the distance of the point of light and the frames is characteristic of verbal reports of the distance of objects (Gogel, 1968).

\section{DISCUSSION}

Presentation of Single Frames

Consider the results in Table 1 obtained from presenting the single frame at the near $(3 \mathrm{ft})$ distance $\left(\mathrm{L}_{\mathrm{N}}\right.$ or $S_{N}$ ). In these situations, the point of light was either at the distance of the frame or was located stereoscopically and perceptually at some distance behind the frame. It will be noted from the medians that positioning the point of light increasingly far behind the frame resulted in either a constant or a decreasing magnitude of induced motion and, as expected, the direction of induced motion almost always was

Table 2

Means, Medians, and Standard Deviations in Inches of Induced Movement of a Point of Light at the Near (N), Middle (M), and Far (F) Distance with the Two Frames, Large (L) and Small (S), Presented Simultaneously, One at the Near and the Other at the Far Distance*

\begin{tabular}{|c|c|c|c|c|c|c|}
\hline & \multicolumn{3}{|c|}{$\begin{array}{c}\text { Large Frame Near, } \\
\text { Small Frame Far }\left(L_{N}, S_{F}\right) \\
\text { Distance of Point of Light }\end{array}$} & \multicolumn{3}{|c|}{$\begin{array}{c}\text { Small Frame Near, } \\
\text { Large Frame } F \text { ar }\left(S_{N}, L_{F}\right) \\
\text { Distance of Point of Light }\end{array}$} \\
\hline & $\mathbf{N}$ & $\mathbf{M}$ & $\mathbf{F}$ & $\mathbf{N}$ & M & $\mathbf{F}$ \\
\hline Mean & +0.34 & +0.85 & +0.45 & -0.09 & +0.10 & +0.63 \\
\hline Median & -0.25 & 0.00 & 0.00 & 0.00 & +0.19 & +0.50 \\
\hline SD & 3.60 & 5.24 & 1.85 & 1.48 & 1.23 & 1.17 \\
\hline Number of + & 7 & 18 & 17 & 17 & 26 & 35 \\
\hline Number of - & 29 & 10 & 7 & 15 & 13 & 6 \\
\hline Number of 0 & 12 & 20 & 24 & 16 & 9 & 7 \\
\hline Average Rank & \multicolumn{3}{|c|}{$\chi^{2} r=19.9, p<.001$} & \multicolumn{3}{|c|}{$\chi^{2} r=24.0, p<.001$} \\
\hline
\end{tabular}

*A positive or negative sign for the mean or median indicates that the induced movement of the point of light was 180 deg out of phase with the physical movement of the far 
$180 \mathrm{deg}$ out of phase with the frame. As suggested by the decrease in the average rank from distances $N$ to $F$ there was some tendency for the induced motion of the point of light to decrease somewhat with increasing distance behind the frame. Consider the results obtained from presenting the single frame at the far $(6 \mathrm{ft})$ distance ( $L_{F}$ or $\left.S_{F}\right)$. In these situations, the point of light was either at the distance $(6 \mathrm{ft})$ of the frame or was located stereoscopically at some distance in front of the frame. As is indicated by the means, medians, and ranks, the magnitude of the induced movement of the point of light clearly decreased with increasing displacement of the point of light from the plane of the frame toward 0 . In general, the consistency and proportion of the change in the magnitude of the induced motion with increasing distance from the frame was greater when the point of light was displaced in depth in front of the far frames than when the point of light was displaced in depth behind the near frames. These results are consistent with those obtained in the previous study by Gogel and Koslow (1971). It ought also to be noted that an appreciable number of reports of no apparent movement of the points of light occurred, particularly when the small frames were used. Possibly, since the angular size of the large frames (at either the near or far position) was larger than the angular size of the small frames (at either the near or far position), the large frames tended to produce more instances of induced motion and, at least in terms of the medians, greater magnitudes of induced motion than the smaller frames.

From Table 3, it is clear that the perceived position of the point of light changed with changes in its physical position and that the median reported positions of the point of light was fairly close to the median reported positions of a frame when the point of light was stereoscopically at the distance of the frame. The importance of this result for the experiment is that there is some evidence that it is the apparent adjacency of objects, not their physical or stereoscopic adjacency, that is the important variable in the adjacency principle (Mershon \& Gogel, 1970).

It can be concluded from the portion of the experiment in which the frames were presented one at a time that the magnitude of the induced movement of the point of light varied as a function of the perceived separation between the point of light and the frame. The decrease in the magnitude of the induced movement tended to be
Table 3

Mean and Average Median Reported Distances in Feet for the $N(3 \mathrm{ft}), M$ (4.5 ft), and F (6 ft) Physical Distances of the Point of Light and the $N(3 \mathrm{ft})$ and $F(6 \mathrm{ft}$ ) Physical Distances of the Frame

\begin{tabular}{|c|c|c|c|c|c|}
\hline & \multicolumn{3}{|c|}{ Point of Light } & \multicolumn{2}{|c|}{ Frame } \\
\hline & $\mathbf{N}$ & $\mathbf{M}$ & $\mathbf{F}$ & $\mathbf{N}$ & $\mathbf{F}$ \\
\hline \multirow{3}{*}{$\begin{array}{l}\text { Mean } \\
\text { Average } \\
\text { Median }\end{array}$} & 3.7 & $\begin{array}{l}\text { One } \\
8.3\end{array}$ & $\begin{array}{c}\text { Frame } \\
13.6\end{array}$ & $\begin{array}{c}\text { Only } \\
\mathbf{3 . 5}\end{array}$ & 6.3 \\
\hline & 2.1 & 3.2 & 4.8 & 1.8 & 4.2 \\
\hline & Two & Fran & nes Sim & ultane & usly \\
\hline \multirow{2}{*}{$\begin{array}{l}\text { Mean } \\
\text { Average } \\
\text { Median }\end{array}$} & 3.7 & 5.8 & 8.0 & 2.4 & 7.9 \\
\hline & 2.2 & 3.0 & 4.5 & 2.0 & 4.6 \\
\hline
\end{tabular}

proportionately greater as the point of light was positioned in front of rather than behind the plane of the frame. This result supports the application of the adjacency principle to induced movement and provides additional evidence for the interpretation of the results in terms of a combination of the adjacency principle and the application of the size-distance invariance hypothesis.

\section{Simultaneous Presentations of Two Frames}

The results from the situations in which two frames moving $180 \mathrm{deg}$ out of phase were presented at the two distances provide rather striking support for the application of the adjacency principle to induced movement. The adjacency principle predicts and the results indicate an increase in the relative contribution to the induced movement of a particular frame as the point of light is closer in apparent depth to this frame and is farther in apparent depth from the other frame. Consider the changes in the median reports of the magnitude of induced movement and the changes in the average ranks as a function of the position of the point of light. These values increase in a positive direction (indicating an increase in the contribution of the far frame to the induced movement) as the point of light was moved from the near to the far position. The means of Table 2 show the same trend for the $S_{N}, L_{F}$ situation but not for the $\mathrm{L}_{\mathrm{N}}, \mathrm{S}_{\mathrm{F}}$ situation. This discrepancy is removed, however, if the scores from one $\mathrm{O}$ are omitted from the results of the $\mathrm{L}_{\mathrm{N}}, \mathrm{S}_{\mathrm{F}}$ situation, with the resulting means $-.161,+.077$, and +.425 for the $N, M$, and $F$ positions of the point of light, respectively. Also consistent with the application of the adjacency principle to induced motion, it will be noted that for both the $L_{N}, S_{F}$ and $S_{N}, L_{F}$ situations the number of positive responses relative to the number of negative responses increased as the point of light was moved from the near to the far position. Clearly, the relative contribution of the near frame to the induced movement of the point of light (negative means or medians) increased as the point of light was increasingly adjacent in depth to the near frame, and the relative contribution of the far frame (positive means or medians) increased as the point of light was increasingly adjacent in depth to the far frame.

The results, shown in Table 2 , from the simultaneous presentations of the two frames demonstrate that the contribution of a frame in the pair of frames to the induced motion increased as the point of light was positioned closer in depth to that frame. But, even though the induced motion of the point of light tended to be determined by the frame that was stereoscopically equidistant with the point of light, the other stereoscopically displaced frame still had an appreciable influence upon the induced motion. It will be recalled that the two simultaneously presented frames were always moving in physically opposite directions (in opposite phase). If depth adjacency were not a factor in the induced movement, the magnitude of the induced motion and the average rank in Table 2 would have been constant (with the average rank always equal to 2.00) for the different distance positions of the light. On the other hand, if the induced motion had been determined completely by the frame with which the point of light was stereoscopically equidistant, the average rank of the near, middle, and far positions of the light in Table 2 would have been $1.00,2.00$, and 3.00 , respectively. The mean of the average ranks of Table 2, combining the results from $L_{N}, S_{F}$ and $S_{N}, L_{F}$, is $1.51,2.03$, and 2.46 for the near, middle, and far distances of the light. It will be noted that the obtained rank of 2.03 for the middle distance is very close to the value predicted from either of the above two possibilities. The obtained ranks of 1.51 and 2.46 are about midway between the values expected from the notion that the frame at the same plane as the light completely determined the induced motion and the notion that the effect of the displaced and equidistant frame is the same. Clearly, although the frame in the same plane as the point of light contributes more to the induction than does the displaced frame, the contribution of the displaced frame under these circumstances remains substantial. Also, it seems that for some Os, a particular frame tends to be dominant for all positions of the point of light, even though this dominance is usually reduced by the depth displacement of the point of 
light from that frame. Such dominance occurred more frequently in the $S_{N}, L_{F}$ situation than in the $\mathrm{L}_{\mathrm{N}}, \mathrm{S}_{\mathrm{F}}$ situation, as is indicated by the larger proportion of + responses in Table 2 from the former as compared with the latter situation for the near position of the point of light. In general, the pattern of results in both Tables 1 and 2 is consistent with the conclusion that superposed upon the adjacency effects is a tendency for the retinally larger or more distant frames (frames with a greater physical but equal retinal movement) to be more effective in producing an induced motion of the point of light.

The amount of induced movement of the point of light attributable to the frame perceptually at the distance of the point of light usually is less in Table 2 than in Table 1. Perhaps this is a consequence of $O$ 's more accurately perceiving the physical movement of this frame as a result of the presence of the second frame. Since Os did not report on the apparent movement of the frames, this possibility cannot be evaluated in the present study. It should be noted, however, that the results from the present study indicate that although depth separation decreased the induction, some amount of induction occurred between objects displaced in depth. If the latter aspect of this conclusion can be applied between frames as well as between a point of light and a frame, the consequence would be that the physical movement of a frame would be more accurately perceived when both frames, rather than the single frame, were present. Thus, induction effects could contribute to veridical perception of movement as well as produce an illusory perception of motion depending upon the physicai motions involved. If correct, this would suggest that induction effects are involved in all perceptions of motion, whether veridical or illusory, when more than one object is present in the visual field.

Although a frame displaced in depth from the point of light had a significant effect upon the induced movement with the induced movement usually determined mainly by the frame equidistant with the point of light, it should not be concluded that a moving frame completely adjacent to a point of light would not completely determine the induced motion regardless of other moving frames in the visual field. It has been found that directional as well as depth separation is an important variable in induction effects (see Gogel, 1972; Mershon, 1972). Although stereoscopic equidistance often eliminated a difference in perceived depth between the frame and point of light, it did not reduce the frontoparallel (directional) separation between these objects. Thus, an appreciable amount of spatial separation between the point of light and any frame necessarily remained for all of the conditions of the experiment.

\section{Implications of the} Adjacency Effects

From the present and previous study (Gogel \& Koslow, 1971), adjacency (or separation) in depth is an important variable in induced movement. Depth adjacency also has been demonstrated to be important in stereoscopic induction (Gogel, 1972) and in brightness induction or brightness contrast (Gogel \& Mershon, 1969; Mershon, 1972; Mershon \& Gogel, 1970). From the research on brightness induction, there is some evidence that the significant depth factor is perceived depth and that the effect of the perceived depth separation of the induction and test object upon the magnitude of the induction is independent of the particular kind of distance cue used in producing the perceived separation of these objects. Generalizing these results, it can be concluded that, all other factors being equal, objects at the same perceived distance will interact more to produce induction effects than objects perceptually separated in space, with the function relating the magnitude of the induction and the apparent separation independent of the cues by which the apparent separation is produced. One of the consequences of this conclusion is that the magnitude of the induction effects cannot be explained entirely (or perhaps to any extent) by adjacency defined at the retinal (proximal) level of stimulation. For example, in the present study if retinal adjacency had been the significant adjacency factor, the retinally smaller frame in the two-frame situation consistently would have determined the direction and magnitude of the induced motion, regardless of the apparent depth position of the point of light. Clearly, this was not the result obtained. It is likely, however, that if the two frames and the point of light had been at the same distance from $O$, the induced motion (in agreement with the adjacency principle) would have been determined mainly by the retinally smaller (inner) frame (Wallach, 1959). Considering the above generalization, these two kinds of results are not opposed. In the case in which the two frames and the point of light are perceptually equidistant, the perception of the equidistance of these three objects is involved in perceiving a frontoparallel displacement of the point of light from each of the frames. Thus depth perception is as important in this case as in the present study. In other words, although perceived separation is proportional to retinal separation when the objects are perceived to be in the same frontoparallel plane, the perception of depth under these conditions is as essential to induction as it is in the case in which the point of light is at different perceived distances with respect to the two frames. In neither case is retinal separation per se the significant variable.

The specification of the adjacency principle directly in terms of perceived spatial separation rather than indirectly in terms of the specific cues of spatial separation emphasizes the interrelation of perceptual events. In the present study the magnitude and direction of the perceived motion of the point of light (the dependent perception) was affected by the perceived position of the point of light with respect to the induction frames (the independent perception). This is a case of one perception affecting another. Other examples of the interrelation of perceptual events are found in the size-distance and shape-slant invariance hypotheses; in the relation between perceptual grouping and the perceived separation of the columns of the display (Rock \& Brosgole, 1964); in the relation between the perception of stroboscopic motion and the apparent position of the stimuli (Rock \& Ebenholtz, 1962); and in the relation between the apparent duration of spiral aftereffects and the perceived distance of the spiral from $\mathrm{O}$ (Mehling, Collins, \& Schroeder, 1971). These and other examples (see Gogel, 1970) indicate that perceptual interrelations occur in a range of situations. The quantitative expression of the relation between an independent and dependent perception might be called a perceptual equation and is to be contrasted to the approach to perception which determines quantitative relations solely between a perceptual and physical event. The distinction between psychophysical and perceptual equations is not in the need to specify physical stimuli since the specification of physical stimuli are involved in both. For example, in the present study the magnitude and direction of the physical or retinal movement of the frames is obviously basic to the phenomenon of induced motion. The distinction is rather that in addition to physical stimulus events, two different perceptual dimensions also are represented in the perceptual equation, one concerned with the independent and the other with the 
dependent perception. If the concept of perceptual interactions is valid a particular value of the independent perception will produce the same effect upon the dependent perception regardless of the cues by which this particular value of the independent perception was obtained. Thus, the identification of perceptual interactions can result in considerable predictive parsimony. In addition, the notion that the consequences of an independent perception for a dependent perception can be specified without regard to the origins of the independent perception suggests a parsimony in the manner in which the observer processes information. If such perceptual interactions result in predictive parsimony, this can occur only because the observer has processed the dependent perception in terms of the independent perception.

From the present study, from other research concerned with the adjacency principle, and from some of the additional evidence cited above, perceived spatial separation is probably the most important of the independent perceptions in that a variety of other perceived characteristics (such as size, color, and shape) seem to depend upon this dimension. Furthermore, the perception of depth is involved ubiquitously in all cases of perceived separation including the special case of perceived frontoparallel displacement. It is as though the magnitudes of dependent perceptions are predictable only as these perceptions become organized in a three-dimensional schema.

\section{REFERENCES}

DUNCKER, $K$. The influence of past experience upon perceptual properties. American Journal of Psychology, 1939, 52, 255-265.

GOGEL, W. C. The effect of set on perceived egocentric distance. Acta Psychologica, 1968, 28, 283-292.

GOGEL, W. C. Depth adjacency and cue effectiveness. Journal of Experimental Psychology, 1972, in press.

GOGEL, W. C., \& KOSLOW, M. The effect of perceived distance on induced movement. Perception \& Psychophysics $1971,10,142-146$.

GOGEL, W. C., \& MERSHON, D. H. Depth adjacency in simultaneous contrast. Perception \& Psychophysics, 1969, 5, 13-17.

MEHLING, K. D., COLLINS, W. E, \& SCHROEDER, D. J, The spiral aftereffect: III. Some effects of perceived size, retinal size and retinal speed on the duration of illusory motion. F.A.A Office of Aviation Medicine Report No. FAA-AM-71-31, 1971.

MERSHON, D. H. Relative contributions o depth and directional adjacency tc whiteness contrast under two conditions of luminance. Vision Research, 1972, in

MERSHON, D. H., \& GOGEL, W. C.Th effect of stereoscopic cues on perceive whiteness. American Journal o Psychology, 1970, 83, 55-67.

ROCK, I., \& BROSGOLE, L. Groupin based on phenomenal proximity. Journa of Experimental Psychology, 1964, 67 $531-538$.

ROCK, I., \& EBENHOLTZ, S. Stroboscopi movement based on change phenomenal rather than retinal location American Journal of Psychology, 1962 75, 193-207.

SIEGEL, S. Nonparame tric statistics for th behavioral sciences. New York McGraw-Hill, 1956.

WALLACH, $H$. The perception of motior Scientific American, 1959, 201, 56-60.

(Received for publication August 13, 1971.) 